Document downloaded from:

http://hdl.handle.net/10251/99668

This paper must be cited as:

Bader, P.; Blanes Zamora, S. (2011). Fourier methods for the perturbed harmonic oscillator in linear and nonlinear Schrödinger equations. Physical Review E. 83(4):46711-1-46711-11. doi:10.1103/PhysRevE.83.046711

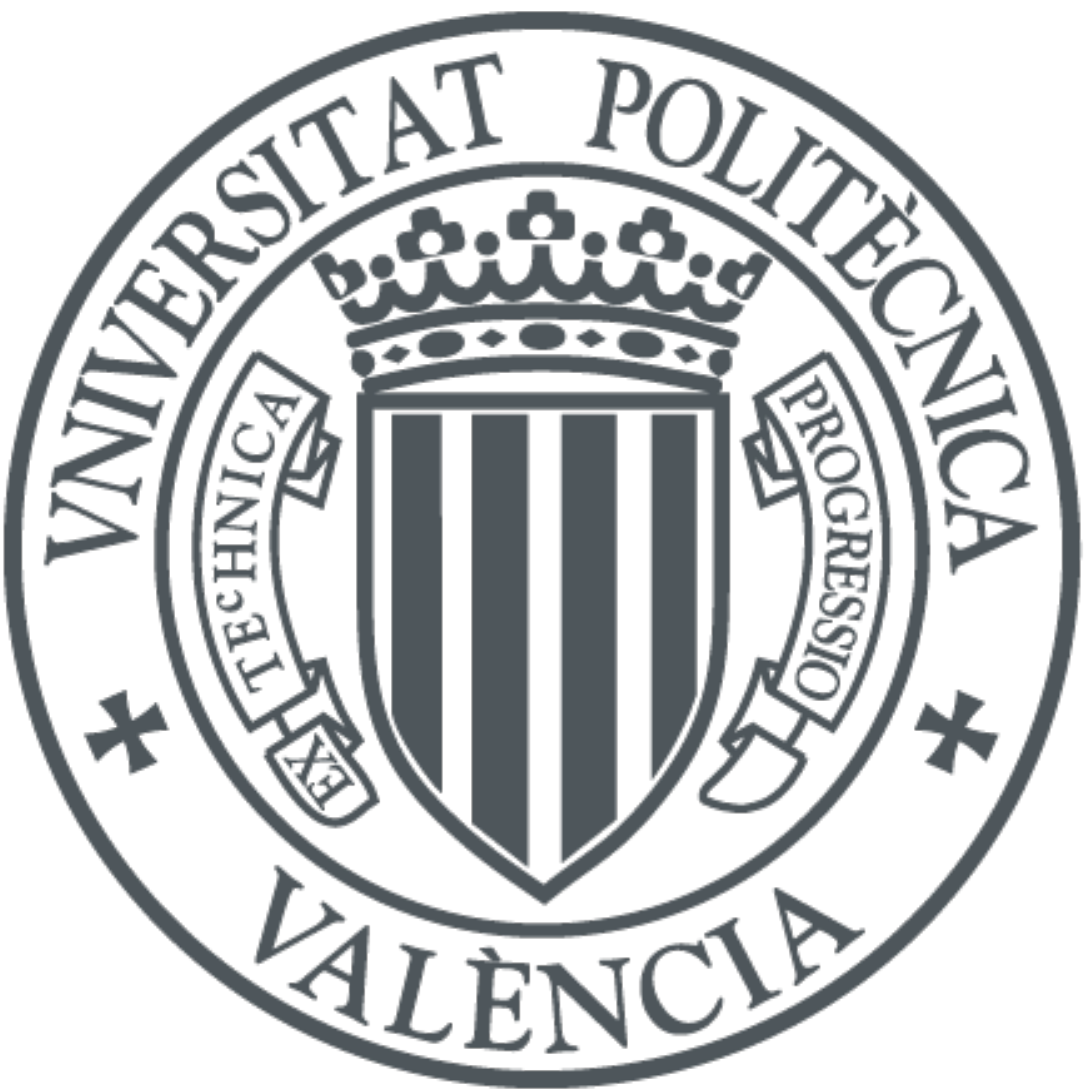

The final publication is available at

http://doi.org/10.1103/PhysRevE.83.046711

Copyright American Physical Society

Additional Information 


\title{
Fourier methods for the perturbed harmonic oscillator in linear and nonlinear Schrödinger equations
}

\author{
Philipp Bader $\circledast$ and Sergio Blanes \\ Universitat Politècnica de València, Instituto de Matemática Multidisciplinar, E-46022 Valencia, Spain
}

\begin{abstract}
We consider the numerical integration of the Gross-Pitaevskii equation with a potential trap given by a time-dependent harmonic potential or a small perturbation thereof. Splitting methods are frequently used with Fourier techniques since the system can be split into the kinetic and remaining part, and each part can be solved efficiently using Fast Fourier Transforms. To split the system into the quantum harmonic oscillator problem and the remaining part allows to get higher accuracies in many cases, but it requires to change between Hermite basis functions and the coordinate space, and this is not efficient for time-dependent frequencies or strong nonlinearities. We show how to build new methods which combine the advantages of using Fourier methods while solving the timedependent harmonic oscillator exactly (or with a high accuracy by using a Magnus integrator and an appropriate decomposition).
\end{abstract}

PACS numbers: 02.60.-x, 02.60.Cb, 02.60.Lj, 02.70.Hm

\section{INTRODUCTION}

The numerical integration of the Gross-Pitaevskii equation (GPE)

$$
i \frac{\partial}{\partial t} \psi(x, t)=\left(-\frac{1}{2 \mu} \Delta+V(x, t)+\sigma(t)|\psi(x, t)|^{2}\right) \psi(x, t)
$$

$x \in \mathbb{R}^{d}$, describing the ground state of interacting bosons at zero temperature, the Bose-Einstein condensates, has attracted great interest [1 3 ] $]$ after the first experimental realizations [4]. We present a new efficient way to solve a special class of GPE, namely that of weakly interacting bosons in a single time-dependent trap. To be more specific, the potential trap $V$ is taken to be a perturbation of the (time-dependent) $d$-dimensional harmonic oscillator, i.e. $V(x, t)=x^{T} M(t) x+\epsilon V_{I}(x, t)$ where $M(t) \in \mathbb{R}^{d \times d}$ is a positive definite matrix and $\epsilon V_{I}(x, t)$ is a small perturbation. The real scalar function $\sigma$ originates from the mean-field interaction between the particles and corresponds to repulsive or attractive forces for positive or negative values of $\sigma(t)$, respectively [5]. Notice that the non-interacting case, $\sigma \equiv 0$, corresponds to the linear Schrödinger equation.

Several methods have been analyzed to compute both the time evolution and the ground state of the GPE in the course of the last decade [1 $3,6,6$, 7], among them finite differences, Galerkin spectral methods and pseudospectral methods for Fourier or Hermite basis expansions. It has been concluded [2] that these pseudospectral methods perform best for a wide parameter range for the GPE. The Fourier type methods can be implemented with Fast Fourier Transform (FFT) algorithms since the trapping $V$ causes the wave function to vanish asymptotically thus allowing to consider the problem as periodic on a sufficiently large spatial interval. Their advantages are high

\footnotetext{
* phiba@imm.upv.es

† serblaza@imm.upv.es
}

accuracy with a moderate number of mesh points and low computational cost. For harmonic oscillator (HO) problems, however, the exact solution is known and by expanding the solution in Hermite polynomials, highly accurate results are obtained if the $\mathrm{HO}$ is solved separately $[2,3,6,6]$.

It is claimed [2], that either Hermite or Fourier pseudospectral methods are the most efficient, the choice depending on the particular parameter set. Motivated by these results, we show how both methods are combined to retain both the accuracy of the Hermite method and the speed of the Fourier transforms, i.e. to rewrite the Hermite method as a single simple pseudospectral Fourier scheme. We have found, that this approximation performs, for the studied problem class, always equal to or better than the original Fourier method and therefore has to compete with Hermite expansions only. Hermite schemes suffer from large computational costs when the number of basis terms in the expansions is altered along the integration or taken very large, being the case for time dependent trap frequencies $M(t)$ or strong nonlinearities $\sigma(t)$. It is in this setup, where our new method substantially improves the Hermite performance, and it can indeed be regarded as the optimal choice for the number of Hermite basis functions (for an equidistant grid) at each time step.

For the ease of notation, we restrict ourselves to the one-dimensional problem

$$
i \frac{\partial}{\partial t} \psi=H_{0}(t) \psi+\left(\varepsilon V_{I}(x, t)+\sigma(t)|\psi|^{2}\right) \psi
$$

where

$$
H_{0}(t)=\frac{1}{2 \mu} p^{2}+\frac{1}{2} \mu \omega^{2}(t) x^{2}
$$

and $p=-i \frac{\partial}{\partial x}$. The boundary conditions imposed by the trap require the wave function to go to zero at infinity, and up to any desired accuracy, we can assume $\psi(x, t)$ and all its derivatives to vanish outside a finite region, say 
$[a, b]$, which we divide using a mesh (usually with $N=$ $2^{k}$ points to allow a simple use of the FFT algorithms). Then, the partial differential equation (I.1) transforms into a system of ordinary differential equations (ODEs)

$$
i \frac{d}{d t} u(t)=\hat{H}_{0}(t) u(t)+\left(\varepsilon V_{I}(X, t)+\sigma(t)|u(t)|^{2}\right) u(t),
$$

and the harmonic part becomes

$$
\hat{H}_{0}(t)=T+\frac{1}{2} \mu \omega^{2}(t) X^{2}
$$

with $u \in \mathbb{C}^{N}$, where $u_{i}(t) \simeq \psi\left(x_{i}, t\right), x_{i}=a+i h, \quad i=$ $0,1, \ldots, N-1, h=(b-a) / N, X=\operatorname{diag}\left\{x_{0}, \ldots, x_{N-1}\right\}$ and $T$ denotes a discretization of the kinetic part.

This system of nonlinear ODEs can be numerically solved by standard all purpose ODE methods. However, because of the particular structure of this problem, different numerical methods can differ considerably in accuracy as well as in computational cost and stability. In addition, the structural properties of the system lead to the existence of several preserved quantities like the norm and energy (for the autonomous case).

The accurate preservation of these quantities as well as the error propagation and performance of splitting methods explain why they are frequently recommended for the time integration [2, 3, 6] and make them subject of investigation in this work.

\section{SPLITTING METHODS}

Let us consider the separable system of ODEs

$$
u^{\prime}=A(u)+B(u), \quad u\left(t_{0}\right)=u_{0} \in \mathbb{C}^{N},
$$

where we assume that both systems

$$
u^{\prime}=A(u), \quad u^{\prime}=B(u)
$$

can either be solved in closed form or accurately integrated. If $\varphi_{t}^{[A]}, \varphi_{t}^{[B]}$ represent the exact flows associated to (II.2) then, to advance the solution one time step, $h$, we can use, for example, the composition $\psi_{h}^{[1]}=\varphi_{h}^{[A]} \circ \varphi_{h}^{[B]}$ (i.e. $\left.u\left(t_{0}+h\right) \simeq \psi_{h}^{[1]}\left(u_{0}\right)=\varphi_{h}^{[A]}\left(\varphi_{h}^{[B]}\left(u_{0}\right)\right)\right)$, which is known as the first-order Lie-Trotter method. A method has order $p$ if $\psi_{h}^{[p]}=\varphi_{h}+\mathcal{O}\left(h^{p+1}\right)$ where $\varphi_{t}$ denotes the exact global flow of (II.1). Sequential application of the two first-order methods $\psi_{h}^{[1]}$ and its adjoint $\psi_{h}^{[1] *}=$ $\varphi_{h}^{[B]} \circ \varphi_{h}^{[A]}$ with half time step yields the second order time-symmetric methods

$$
\begin{aligned}
& \psi_{h, A}^{[2]}=\varphi_{h / 2}^{[A]} \circ \varphi_{h}^{[B]} \circ \varphi_{h / 2}^{[A]} \\
& \psi_{h, B}^{[2]}=\varphi_{h / 2}^{[B]} \circ \varphi_{h}^{[A]} \circ \varphi_{h / 2}^{[B]}
\end{aligned}
$$

(referred as $A B A$ and $B A B$ compositions). The contraction via the (1-parameter-)group property of the flows that eliminated one computation is called First Same As Last (FSAL) property and can also be used with higher order $m$-stage compositions

$$
\psi_{h}^{[p]}=\varphi_{a_{m} h}^{[A]} \circ \varphi_{b_{m} h}^{[B]} \circ \cdots \circ \varphi_{a_{1} h}^{[A]} \circ \varphi_{b_{1} h}^{[B]}
$$

if $a_{m}=0$ or $b_{m}=0$ and repeated application of the scheme without requiring output. For linear problems, it is usual to replace the flow-maps by exponentials (for nonlinear problems the same is possible using exponentials of Lie operators). In this notation, the equation $i u^{\prime}=A(u)+B(u)$, whose formal solution for the evolution operator is denoted by $\phi_{t}^{[A+B]}=e^{-i t(A+B)}$, is approximated for one time step, $h$, by the order $p$ composition (II.5) or, equivalently,

$$
\psi_{h}^{[p]} \equiv e^{-i h a_{m} A} e^{-i h b_{m} B} \cdots e^{-i h a_{1} A} e^{-i h b_{1} B} .
$$

We keep in mind that, in a nonlinear problem, if $B$ depends on $u$, it has to be updated at each stage because $u$ changes during the evolution of $e^{-i h a_{i} A}$.

Backward error analysis shows that the action of a splitting method is equivalent to solve exactly, for one time step $h$, a perturbed differential equation (for sufficiently small $h$ ) that can contain higher order commutators

$$
\begin{aligned}
u^{\prime} & =\left(A+B+h \alpha_{1,1}[A, B]\right. \\
& \left.+h^{2}\left(\alpha_{2,1}[A,[A, B]]+\alpha_{2,2}[B,[B, A]]\right)+\ldots\right) u
\end{aligned}
$$

( $[A, B]:=A B-B A$, and for simplicity, we have denoted $A=A(u) \cdot \nabla, B=B(u) \cdot \nabla)$ where the coefficients $a_{i}, b_{i}$ are chosen in order to cancel out the coefficients $\alpha_{i, j}$ up to a given order. It is thus important to analyze the dominant contributions to the error from the commutators, in order to choose the most appropriate method or to build new ones.

There exist many different splitting methods which are designed for different purposes, depending on the structure of the problem, the desired order, the required stability, etc. [8 14]. If the operator $A$ corresponds to the kinetic part, which is quadratic in momenta, and the operator $B$ is the potential, diagonal in coordinate space, the commutator $[B,[B,[B, A]]]$ vanishes and partitioned Runge-Kutta-Nyström methods become favorable. The coefficients for this family of methods have to solve a significantly reduced number of order conditions and, in general, their performance is superior to splitting methods designed for general separable problems, in addition to being more stable. Efficient schemes of order 4 and 6 are obtained in [9]. On the other hand, when $H_{0}$ is the dominant part, it is worth to take a closer look at the split (I.1). This would correspond to $\|B\| \ll\|A\|$ and for this case, when facing autonomous problems, there exist tailored methods which have shown a high performance in practice. Writing the equation as $i u^{\prime}=(A+\epsilon B) u$ (with $\epsilon$ a small parameter), it is clear that the local error of the second order methods (II.3) or (II.4) comes from the commutators at third order $([A,[A, \epsilon B]]$ and 
$[\epsilon B,[A, \epsilon B]])$ and we can say that the local error is of order $\mathcal{O}\left(\epsilon h^{3}+\epsilon^{2} h^{3}\right)$. The coefficients $a_{i}, b_{i}$ in the general composition (II.6) can be chosen to cancel the dominant error terms, say, the $\mathcal{O}\left(\epsilon h^{r}\right)$ terms for relatively large values of $r$. Then, one can denote the effective order of a method by $(r, p)$ with $r \geq p$ when the local error is given by $\mathcal{O}\left(\epsilon h^{r+1}+\epsilon^{2} h^{p+1}\right)$. The method is of order $p$, but in the limit $\epsilon \rightarrow 0$ it is considered as of order $r \geq p$. Using this split allows to gain a factor $\epsilon$ in the accuracy even for general splitting methods where $r=p$. In 11], several methods of order $(r, 2)$ for $r \leq 10$ are obtained with all coefficients $a_{i}, b_{i}$ being positive and some other schemes of order $(r, 4)$ for $r=6,8$ are presented. For near-integrable systems, these last methods are the most efficient and stable ones. Despite the gain of accuracy, the split into a dominant part and a small perturbation is left unconsidered when it leads to involved or computationally costly algorithms. This issue is addressed in this work.

To take account for the time dependence in the vector fields in (I.1), a more detailed analysis is required. The general separable equation

$$
i u^{\prime}=A(u, t)+\epsilon B(u, t)
$$

can be solved by considering the time as two new independent coordinates

$$
\left\{\begin{array}{rl}
i u^{\prime} & =A\left(u, t_{2}\right) \\
t_{1}^{\prime} & =1
\end{array}, \quad\left\{\begin{array}{rl}
i u^{\prime} & =\epsilon B\left(u, t_{1}\right) \\
t_{2}^{\prime} & =1
\end{array} .\right.\right.
$$

This standard split is equivalent to a new system in an extended phase space which is not near-integrable and the highly efficient and stable methods designed for those problems lose their excellent performance. Following [15], the near integrability is recovered if we introduce only one variable as follows

$$
\left\{\begin{array}{rl}
i u^{\prime} & =A\left(u, t_{1}\right) \\
t_{1}^{\prime} & =1
\end{array}, \quad i u^{\prime}=\epsilon B\left(u, t_{1}\right) .\right.
$$

This split requires to solve exactly the equation $i u^{\prime}=$ $A(u, t)$ for the corresponding fractional time steps and to freeze the time when solving $i u^{\prime}=\epsilon B\left(u, t_{1}\right)$ (see Table【).

TABLE I. Algorithm for the numerical integration of the system (II.10) by the composition (II.5).

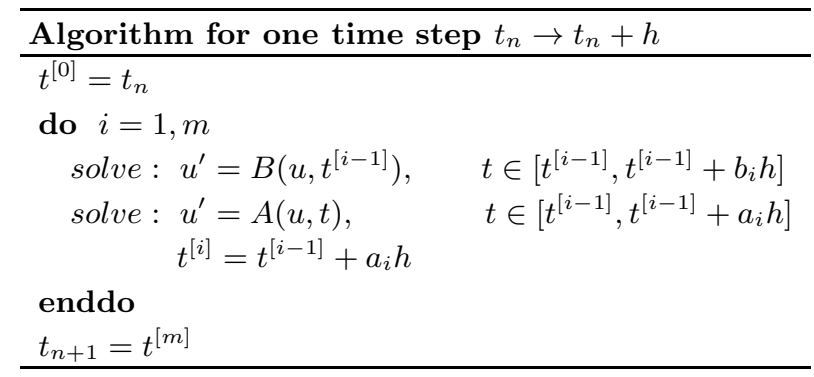

We show that the exact solution of the nonautonomous problem, in our setting the dominant part
$H_{0}$ of (I.1),

$$
i \frac{\partial}{\partial t} \psi=\left(\frac{1}{2 \mu} p^{2}+\frac{1}{2} \mu \omega^{2}(t) x^{2}\right) \psi
$$

is easily computed for a time step using Fourier transforms. Before giving the details on the time integration, some remarks on the formal solution are necessary.

It is well known that $H_{0}$ is an element of the Lie algebra spanned by the operators $\left\{E=x^{2} / 2, F=p^{2} / 2, G=\right.$ $\left.\frac{1}{2}(p x+x p)\right\}$, where $\mu=1$ for simplicity, and its commutators are

$$
[E, F]=i G, \quad[E, G]=2 i E, \quad[F, G]=-2 i F .
$$

This is a three-dimensional Lie algebra and the solution, $\psi(x, t)=U(t, 0) \psi(x, 0)$, of (II.11) can be expressed as a single exponential using the Magnus series expansion 16, 17] or as a product of exponentials [18]. It is possible to formulate the evolution operator $U(t, 0)$ in many different ways, the most appropriate depending on the particular purpose, e.g. using the Magnus expansion

$$
U(t, 0)=\exp \left(f_{1}(t) E+f_{2}(t) F+f_{3}(t) G\right)
$$

for certain functions $f_{i}(t)$ [19]. Approximations of (II.12) for one time step, $h$, on the other hand, are easily obtained, e.g. a fourth-order commutator-free method is given by [20]

$$
\begin{aligned}
U(t+h, t) & =\exp \left(\frac{h}{2}\left(\frac{1}{2} p^{2}+\omega_{L}^{2} \frac{1}{2} x^{2}\right)\right) \\
& \times \exp \left(\frac{h}{2}\left(\frac{1}{2} p^{2}+\omega_{R}^{2} \frac{1}{2} x^{2}\right)\right)+\mathcal{O}\left(h^{5}\right)
\end{aligned}
$$

where

$$
\omega_{L}^{2}=\alpha \omega_{1}^{2}+\beta \omega_{2}^{2}, \quad \omega_{R}^{2}=\beta \omega_{1}^{2}+\alpha \omega_{2}^{2}
$$

with $\omega_{i}=\omega\left(t_{n}+c_{i} h\right), c_{1}=\frac{1}{2}-\frac{\sqrt{3}}{6}, c_{2}=\frac{1}{2}+\frac{\sqrt{3}}{6}$, and $\alpha=\frac{1}{2}-\frac{1}{\sqrt{3}}, \beta=1-\alpha$. It can be considered as the composition of the evolution for half time step of two oscillators with averaged frequencies, using the fourth-order Gauss-Legendre quadrature rule to evaluate $\omega(t)$. Different quadrature rules can also be be used and correspond to different averages along the time step, see [17, 20]. In the limit when $\omega$ is constant the exact solution is recovered. Higher order approximations are available, if more accurate results are desired, by approximating the functions $f_{i}$ in (II.12) via truncated Magnus expansions.

Our objective is to obtain a factorization of the solution which only involves terms proportional to $E$ or $F$ since they are easy to compute as we show in the following paragraph.

Starting from (II.13) or high order approximates of (II.12), the main result of this work is the natural decomposition for the application of Fourier spectral methods. 


\section{A. Hamiltonians for spectral methods}

We now analyze how to compute the evolution of different parts of the Hamiltonian by spectral methods. The spatial derivative (or kinetic part) associated to the semidiscretized problem (I.3) can be solved in the momentum space by noting that

$$
i \frac{d}{d t} u(t)=T u(t)=\mathcal{F}_{N}^{-1} D_{N} \mathcal{F}_{N} u
$$

where $D_{N}$ is diagonal and $\mathcal{F}_{N}$ denotes the discrete Fourier transform of length $N$, whose computation can be accomplished by the FFT algorithm with $\mathcal{O}(N \log N)$ floating point operations. The solution of (II.14) for one time step, $h$, is given by

$$
u(t+h)=\mathcal{F}_{N}^{-1} e^{-i h D_{N}} \mathcal{F}_{N} u(t)
$$

which requires two FFT calls. The exponentials in $e^{-i h D_{N}}$ need to be computed only once and can be reused at each step such that the cost of the action of $e^{-i h D_{N}}$ corresponds to $N$ complex products.

For the remaining part, the following well-known result is very useful

Lemma II.1 If $F$ is real valued, the equation

$$
i \frac{\partial}{\partial t} \phi(x, t)=F(x,|\phi(x, t)|) \phi(x, t)
$$

leaves the norm invariant, $|\phi(x, t)|=|\phi(x, 0)|$, and then

$$
\phi(x, t)=e^{-i t F(x,|\phi(x, 0)|)} \phi(x, 0) .
$$

On the other hand, it is well known that the solutions of the linear Schrödinger equation with the harmonic potential

$$
i \frac{\partial}{\partial t} \phi(x, t)=\frac{1}{2}\left(p^{2}+x^{2}\right) \phi(x, t)
$$

can be expressed in terms of Hermite polynomials:

$$
\phi(x, t)=\sum_{n=0}^{\infty} c_{n} e^{-i E_{n} t} h_{n}(x)
$$

where

$$
E_{n}=n+\frac{1}{2}, \quad h_{n}(x)=\frac{1}{\pi^{1 / 4} \sqrt{2^{n} n !}} H_{n}(x) e^{-x^{2} / 2}
$$

and $H_{n}(x)$ are the Hermite polynomials satisfying the recursion

$$
H_{k+1}(x)=2 x H_{k}(x)-2 k H_{k-1}, \quad k=1,2, \ldots
$$

with $H_{0}(x)=1, H_{1}(x)=2 x$. The weights $c_{n}$ can be computed from the initial conditions, $c_{n}=$ $\int h_{n}(x) \phi(x, 0) d x$.
The previous results show how to compute individual parts of the equation and thus permit different ways of splitting the system in two solvable parts

$$
i \psi_{t}=(A+B) \psi
$$

We consider the following cases:

\section{(i) Fourier(F)-split.}

$$
A=\frac{1}{2} p^{2}, \quad B(t)=\frac{\omega(t)}{2} x^{2}+\varepsilon V_{I}(x, t)+\sigma(t)|\psi|^{2},
$$

We take the time as a new coordinate, as in (II.10), and evolve it with $A$, which is now autonomous and exactly solvable, and freeze the time in $B$, which is then solved using the result from Lemma II.1. Here, $A$ and $B$ are diagonal in the momentum and coordinate spaces, respectively, and we can change between them using the Fourier Transforms.

(ii) Harmonic oscillator(HO)-split. Let for a moment $w=1$, the Hermite expansion then suggests a split

$$
A=\frac{1}{2}\left(p^{2}+x^{2}\right), \quad B(t)=\varepsilon V_{I}(x, t)+\sigma(t)|\psi|^{2},
$$

where the solution for the equation $i u^{\prime}=A u$ can be approximated using a finite number of Hermite basis functions, i.e.

$$
\phi_{M}(x, t)=\sum_{n=0}^{M-1} c_{n} e^{-i E_{n} t} h_{n}(x)
$$

Since $B$ is diagonal in the coordinate space it will act as a simple multiplication if we choose a number of Hermite basis functions and evaluate them at the points of a chosen mesh (e.g. using the Gauss-Hermite quadrature 21] or on equidistant grid points [2]). This split can be of interest if all contributions from $B(t)$ are small with respect to $A$ and methods for near-integrable systems are used. If the frequency is time dependent, the corresponding split is

$$
A(t)=\frac{1}{2}\left(p^{2}+\omega^{2}(t) x^{2}\right), \quad B(t)=\varepsilon V_{I}(x, t)+\sigma(t)|\psi|^{2} .
$$

In this case, it is convenient to take the time, $t$ as a new coordinate as shown in (II.10). The solution of $i u^{\prime}=A(t) u$, in the algorithm in Table 1 can be approximated by the Magnus expansion, e.g. (II.12) or (II.13), but these factorizations are not appropriate for use with spectral methods since it would require two sets of basis functions and additional transformations.

In general, the split (i) can be considered faster and simpler since $A \equiv T$ can be computed in the momentum space, and one can easily and efficiently change from momentum to coordinate space via FFTs. The choice (ii), on the other hand, allows us to take advantage of the structure of a near-integrable system if, roughly speaking, $\|B\|<\|A\|$, but it requires to solve the equation 
for the (time-dependent) harmonic potential exactly (or with high accuracy). The evolution of the constant oscillator is easily computed using Hermite polynomials (see $[2,[3,21]$ ). As mentioned, methods tailored for this family of problems show a high performance. The main drawback, however, is that the evolution for $e^{-i h a_{i} A}$ has to be carried out using a basis of Hermite polynomials whereas the $B$ part is advanced in space coordinates [21] resulting in possibly costly basis transformations.

\section{B. Solving the Harmonic oscillator by Fourier methods}

We propose a new method which combines the advantages of both splittings. It retains the advantages of the HO-split (ii) while being as fast to compute as the Fsplit in (i). For this purpose, we briefly review some basic concepts of Lie algebras.

Given $X, Y$ two elements of a given Lie algebra it is well known that

$$
e^{X} Y e^{-X}=Y+[X, Y]+\frac{1}{2}[X,[X, Y]]+\ldots
$$

If $\left\{X_{1}, \ldots, X_{k}\right\}$ are the generators of a finite dimensional Lie algebra and

$$
Z=\sum_{n=1}^{k} \alpha_{n} X_{n}, \quad G=\sum_{n=1}^{k} a_{n} X_{n}
$$

then

$$
G(t)=e^{t Z} G e^{-t Z}=\sum_{n=1}^{k} a_{n}(t) X_{n}
$$

where $G(t)$ satisfies the differential equation

$$
G^{\prime}(t)=[Z, G(t)], \quad G(0)=G .
$$

Notice that if $G=x$ or $G=p$ and $Z=H(x, p)$ these equations are equivalent to the equation of motion for the classical Hamiltonian $H(x, p)$. It is also well-known that two operators are identical on a sufficiently small time interval if they satisfy the same first order differential equation with the same initial conditions [22].

Given $X(x), P(p), W=\frac{1}{2}\left(p^{2}+x^{2}\right)$ and an analytic function $F(x, p)$, we are interested in the following adjoint actions

$$
\begin{aligned}
e^{-i t X} F(x, p) e^{i t X} & =F\left(x, p+t X^{\prime}\right) \\
e^{-i t P} F(x, p) e^{i t P} & =F\left(x-t P^{\prime}, p\right) \\
e^{-i t W} F(x, p) e^{i t W} & =F(\hat{x}, \hat{p})
\end{aligned}
$$

where $X^{\prime}=d X / d x, P^{\prime}=d P / d p$ and

$$
\begin{aligned}
& \hat{x}=\cos (t) x+\sin (t) p \\
& \hat{p}=-\sin (t) x+\cos (t) p .
\end{aligned}
$$

In classical mechanics, this corresponds to a kick, a drift and the harmonic oscillator rotation.

As we have seen, the exponentials can be easily computed by Fourier spectral methods. It is then natural to ask the question if it is possible to write the solution of (II.11) as a product of exponentials which are solvable by spectral methods. The answer is positive and it is formulated as follows.

Let us first consider the pure harmonic oscillator, whose result was obtained in [23], and for which we present a new proof that applies equally to the general case.

Lemma II.2 Let $A_{1}=\frac{1}{2} p^{2}, B_{1}=\frac{1}{2} x^{2}$ and

$$
g(t)=\sin (t), \quad f(t)=\tan (t / 2) .
$$

Then, the following property is satisfied for $|t|<\pi$ :

$$
\begin{aligned}
e^{-i t\left(A_{1}+B_{1}\right)} & =e^{-i f(t) A_{1}} e^{-i g(t) B_{1}} e^{-i f(t) A_{1}} \\
& =e^{-i f(t) B_{1}} e^{-i g(t) A_{1}} e^{-i f(t) B_{1}}
\end{aligned}
$$

Proof. It suffices to impose that the right hand side of (II.27) (or (II.28) ) satisfies the equation (II.17) with the identity as initial condition what can be verified by differentiating and simple applications of the rules (II.25a b).

A more constructive way to derive the functions $f, g$ makes use of the parallelism with the one-dimensional classical harmonic oscillator with Hamiltonian $H=$ $\frac{1}{2} p^{2}+\frac{1}{2} q^{2}$ and Hamilton equations

$$
\frac{d}{d t}\left\{\begin{array}{l}
q \\
p
\end{array}\right\}=\left(\begin{array}{cc}
0 & 1 \\
-1 & 0
\end{array}\right)\left\{\begin{array}{l}
q \\
p
\end{array}\right\}=(A+B)\left\{\begin{array}{l}
q \\
p
\end{array}\right\}
$$

where

$$
A \equiv\left(\begin{array}{cc}
0 & 1 \\
0 & 0
\end{array}\right), \quad B \equiv\left(\begin{array}{cc}
0 & 0 \\
-1 & 0
\end{array}\right) .
$$

The Lie algebra generated by the matrices $A, B$ is the same as the Lie algebra associated to the operators $A_{1}, B_{1}$ for the Schrödinger equation with the harmonic potential (II.17).

The exact evolution operator of (II.29) is

$$
O(t)=\left(\begin{array}{rr}
\cos (t) & \sin (t) \\
-\sin (t) & \cos (t)
\end{array}\right)
$$

which is an orthogonal and symplectic $2 \times 2$ matrix. For the splitted parts, the solutions are easily computed to

$$
e^{f(t) A}=\left(\begin{array}{cc}
1 & f(t) \\
0 & 1
\end{array}\right), \quad e^{g(t) B}=\left(\begin{array}{cc}
1 & 0 \\
-g(t) & 1
\end{array}\right)
$$

and then, equating the symmetric composition

$$
e^{f A} e^{g B} e^{f A}=\left(\begin{array}{cc}
1-f \cdot g & 2 f-f^{2} \cdot g \\
-g & 1-f \cdot g
\end{array}\right),
$$


to (II.31), we obtain (II.26) which is valid for $|h| \leq \pi$. The decomposition (II.28) is derived analogously. Using the Baker-Campbell-Haussdorf-formula, it is clear, that both results remain valid, up to the first singularity at $t= \pm \pi$, when replacing the matrices $A, B$ by the corresponding linear operators $A_{1}, B_{1}$, since all computations are done in identical Lie algebras.

It is immediate to generalize this result to the equation

$$
i \frac{\partial}{\partial t} \psi(x, t)=\left(\frac{1}{2 \mu} p^{2}+\mu \frac{\omega^{2}}{2} x^{2}\right) \psi(x, t),
$$

$\mu, \omega>0$ by replacing (II.26) with

$$
g=\frac{1}{\mu \omega} \sin (\omega t), \quad f=\mu \omega \tan \left(\frac{\omega}{2} t\right) .
$$

This result is valid for $|t|<t^{*} \equiv \pi / \omega$.

The following theorems extend this idea to decompositions of operators that appear after the approximation of the time dependent parts via (II.12) or by the composition (II.13).

Theorem II.3 Let $\alpha, \beta, \gamma$ be constants, $\eta=\sqrt{\alpha \gamma-\beta^{2}}$ and

$$
\begin{aligned}
& g(t)=\gamma / \eta \cdot \sin (\eta t), \\
& f(t)=\frac{1}{g(t)}\left(1-\cos (\eta t)+\frac{\beta}{\eta} \sin (\eta t)\right), \\
& e(t)=\frac{1}{g(t)}\left(1-\cos (\eta t)-\frac{\beta}{\eta} \sin (\eta t)\right) .
\end{aligned}
$$

Then, the following decomposition holds for $0 \leq t<\pi / \eta$ :

$$
\begin{aligned}
& e^{-i \frac{t}{2}\left(\alpha x^{2}+\beta(x p+p x)+\gamma p^{2}\right)} \\
= & e^{-i f(t) \frac{1}{2} x^{2}} e^{-i g(t) \frac{1}{2} p^{2}} e^{-i e(t) \frac{1}{2} x^{2}} .
\end{aligned}
$$

Proof. The proof follows the lines of the proof of Lemma 【I.2. The evolution operator associated to the classical Hamiltonian $H=\frac{1}{2}\left(\alpha x^{2}+2 \beta x p+\gamma p^{2}\right)$ is given by

$$
\left(\begin{array}{cc}
\cos (\eta t)+\frac{\beta}{\eta} \sin (\eta t) & \frac{\gamma}{\eta} \sin (\eta t) \\
-\frac{\alpha}{\eta} \sin (\eta t) & \cos (\eta t)-\frac{\beta}{\eta} \sin (\eta t)
\end{array}\right) .
$$

and equality to the right hand side of (II.33) is verified by straight-forward computation of the matrix exponentials. The solution is valid until the first singularity at $t=\pi / \eta$. Using (II.25a b), it can be checked that both sides of (II.33) satisfy the same differential equation and initial conditions.

Theorem II.4 Let $\omega_{k} \in \mathbb{R}, c_{k}=\cos \left(\omega_{k} t / 2\right), s_{k}=$ $\sin \left(\omega_{k} t / 2\right)$ for $k=L, R$ and

$$
\begin{aligned}
& g(t)=s_{L} c_{R} / \omega_{L}+c_{L} s_{R} / \omega_{R}, \\
& f(t)=\frac{1}{g(t)}\left(1-c_{L} c_{R}+\frac{\omega_{L}}{\omega_{R}} s_{L} s_{R}\right), \\
& e(t)=\frac{1}{g(t)}\left(1-c_{L} c_{R}+\frac{\omega_{R}}{\omega_{L}} s_{L} s_{R}\right) .
\end{aligned}
$$

Then, the following decomposition

$$
\begin{aligned}
& e^{-i \frac{t}{2}\left(\frac{1}{2} p^{2}+\omega_{L}^{2} \frac{1}{2} x^{2}\right)} e^{-i \frac{t}{2}\left(\frac{1}{2} p^{2}+\omega_{R}^{2} \frac{1}{2} x^{2}\right)} \\
= & e^{-i f(t) \frac{1}{2} x^{2}} e^{-i g(t) \frac{1}{2} p^{2}} e^{-i e(t) \frac{1}{2} x^{2}}
\end{aligned}
$$

is satisfied for $0 \leq t<t^{*}$, where $t^{*}$ is the smallest positive root of $g(t)$.

The proof is similar to the previous one.

\section{THE HERMITE-FOURIER METHODS}

With the presented exact decompositions at hand, we now solve the discretized GPE (I.3) by splitting methods using the symmetric compositions (II.3) and (II.4). Let us first consider the case $w=1$ and take the HO split $A=A_{1}+B_{1}$

$$
\begin{aligned}
& \psi_{h, A}^{[2]}=e^{-i h\left(A_{1}+B_{1}\right) / 2} e^{-i h B} e^{-i h\left(A_{1}+B_{1}\right) / 2} \\
& \psi_{h, B}^{[2]}=e^{-i h B / 2} e^{-i h\left(A_{1}+B_{1}\right)} e^{-i h B / 2} .
\end{aligned}
$$

Replacing the exponentials $e^{-i h\left(A_{1}+B_{1}\right)}$ by (II.27) or (II.28), we obtain four different methods whose computational costs differ considerably. At first sight, using the FSAL property, both (III.1) and (III.2) are equivalent from the computational point of view and require one exponential of $B$ and another one of $A_{1}+B_{1}$ per step. However, a significant difference arises when we plug in the decompositions (II.27) or (II.28). Only the combination (III.2) with (II.28) yields a method that involves only a single FFT call per step

$$
\begin{aligned}
\psi_{h}^{[2]} & =e^{-i h B / 2} e^{-i h\left(A_{1}+B_{1}\right)} e^{-i h B / 2} \\
& =e^{-i h B / 2} e^{-i f(h) B_{1}} e^{-i g(h) A_{1}} e^{-i f(h) B_{1}} e^{-i h B / 2} \\
& =e^{-i\left(h B / 2+f(h) B_{1}\right)} e^{-i g(h) A_{1}} e^{-i\left(h B / 2+f(h) B_{1}\right)}
\end{aligned}
$$

and solves exactly the harmonic oscillator for $|h|<h^{*}$. For any other combination, more kinetic terms have to be computed per step since the FSAL property cannot be exploited to full extent and hence result in more costly 24] methods for the same accuracy.

The general composition (II.6) can be rewritten in the same way by replacing each flow $e^{-i a_{i} A}$ in (II.6) by the composition (II.28)

$$
\begin{aligned}
\Phi_{h} \equiv & e^{-i\left(h b_{m} B+\alpha_{m} B_{1}\right)} e^{-i g\left(a_{m} h\right) A_{1}} e^{-i\left(h b_{m} B+\alpha_{m-1} B_{1}\right)} \\
& \cdots e^{-i\left(h b_{1} B+\alpha_{1} B_{1}\right)} e^{-i g\left(a_{1} h\right) A_{1}} e^{-i \alpha_{0} B_{1}}
\end{aligned}
$$

where $\alpha_{k}=f\left(a_{k+1} h\right)+f\left(a_{k} h\right), k=0,1, \ldots, m+1$ with $a_{0}=a_{m+1}=0$. This method is valid for $\left|a_{i} h\right|<h^{*}, \quad i=$ $1, \ldots, m$ and requires only $m$ calls of the FFT and its inverse, but reaches the same accuracy as if the Hermite functions were used. 
In the more general case with a time-dependent frequency, $\omega(t)$, starting from the HO splitting, the timedependent part is first approximated by Magnus expansions (II.12) or (II.13). Theorems $\amalg .3$ and $\amalg .4$ then provide decompositions to write the product of exponentials in a similar way as in (III.4) but now $\alpha_{k}=f\left(a_{k+1} h\right)+$ $e\left(a_{k} h\right)$ and it is valid for $\left|a_{i} h\right|<t^{*}, \quad i=1, \ldots, m$ where $t^{*}$ is the first zero of $g(t)$. At each stage, one has to compute $f\left(a_{i} h\right), g\left(a_{i} h\right), e\left(a_{i} h\right)$ from $\omega(t)$.

As in the previous case, it only requires $m$ calls of the FFT and its inverse, like the standard Fourier pseudospectral methods. For stability reasons, it seems convenient to look for splitting method whose value of $\max _{i}\left\{\left|a_{i}\right|\right\}$ is as small as possible.

\section{NUMERICAL EXAMPLES}

We analyze first the performance of the methods considered in this work for the one-dimensional problem (I.1) with $\mu=\omega^{2}=1$, and the pure harmonic trap, i.e. $V_{I}=0$.

To illustrate the validity of the decomposition presented in Lemma II.2, we first consider the linear problem $(\sigma=0)$. We take the ground state at $t=0$ as initial condition whose exact solution is given by

$$
\psi(x, t)=\frac{1}{\pi^{1 / 4} \sqrt{2^{n} n !}} e^{-i t / 2} e^{-x^{2} / 2} .
$$

We discretize on the interval $[-10,10]$, to ensure the wave function and its first derivatives vanish up to round off at the boundaries, and sample it at $N=1024$ equidistant grid points. We integrate, with only one time step, from $t=0$ to $T$ for $T \in[-\pi, \pi]$, i.e. forward and backward in time. We measure the integrated error in the wave function, $\left\|u_{e x}(T)-u_{a}(T)\right\|_{2}$, where $u_{a}(T)$ denotes the approximate numerical solution obtained using the split (II.28) and $u_{e x}(T)$ is the exact solution at the discretized mesh. The result of this comparison is illustrated in Fig. 1 (left). The split (II.28) reproduces, for $|T|<\pi$, the exact solution up to round off, as expected. The right panel in Fig. 1 displays a zoom near a singularity where the error grows rapidly due to double precision arithmetic.

We analyze how the approximation properties of the Hermite decomposition (II.22b strongly depend on the function in question and on the chosen number of basis functions, $M$. We compute the $M$ required to reach round-off precision for the evolution of a displaced ground state as initial condition, $\psi_{\delta}(x, 0)=$ $e^{-(x-\delta)^{2} / 2} /\left(\pi^{1 / 4} \sqrt{2^{n} n !}\right)$ from $t=0$ to $T=10$ in one time step. From initial conditions computed on a mesh, this can be accomplished as follows [2]:

$$
u_{e x}(T)=e^{-i T\left(A_{1}+B_{1}\right)} u_{0} \sim K^{T} e^{-i T D_{1}} K u_{0}
$$

where $D_{1}=\operatorname{diag}\left\{\frac{1}{2}, \frac{3}{2}, \ldots, \frac{2 M-1}{2}\right\}, M$ is the number of basis elements considered, and $K_{i, j}=h_{i-1}\left(x_{j}\right), i=$

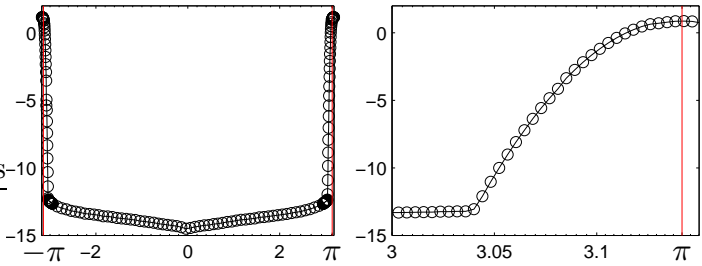

FIG. 1. Error in logarithmic scale for the integration of the ground state of the Harmonic potential using the split (II.28) for $T \in[-\pi, \pi]$ (integration forward and backward in time). The left and right panels show the 2-norm error and a zoom about $T=\pi$, respectively.

$1, \ldots, M, j=1, \ldots, N=512$ with $h_{n}(x)$ given in (II.18), $x \in[-10,10]$. For $\delta=\frac{1}{10}$, round off accuracy is achieved with $M=8$ while for $\delta=2$ it is necessary to take $M=29$. We observe that the Hermite decomposition is very sensitive to the initial conditions [21]. The Hermite basis works efficiently as far as the initial conditions as well as the evolution thereof can be accurately approximated using a few number of basis elements and one has to keep in mind that, for nonlinear problems, the number of basis functions necessary to reach a given accuracy can vary along the time integration.

Next, we study the following values for the nonlinearity parameter: $\sigma=10^{-2}, 1,10^{2}$. The case $\sigma=10^{-2}$ illustrates the performance of the new methods if applied to problems (linear or nonlinear) which are small perturbations of the Harmonic potential whereas the values $\sigma=1,10^{2}$ are large enough to demonstrate the nonlinearity effects on the approximation properties of the methods. Physically, $\sigma$ is proportional to the number of particles in a Bose-Einstein condensate and to the interaction strength [5].

For all cases, we choose the initial condition $\psi(x, 0)=$ $\rho e^{-(x-1)^{2} / 2}$, with $\rho$ a normalizing constant. We show in Fig. 2 the value of $|\psi(x, t)|^{2}$ at the initial and final time. The spatial interval is adjusted to ensure the wave function vanishes (up to round off) at the boundaries, here $[-20,20]$ for $\sigma=0.01$ and $[-30,30]$ for both $\sigma=1$ and $\sigma=100$ where the wave function moves faster (we only show the interval $x \in[-5,5])$. One can appreciate that for strong nonlinearities the wave function can considerably penetrate the potential barrier and one expects that an accurate approximation of these wave functions requires a large number of Hermite functions when using (IV.1) and hence renders this procedure inappropriate.

\section{A. HO-split versus F-split}

We analyze now the advantages of the HO-split versus the F-split as given in (II.23) and (II.21).

In a first experiment, we fix the symmetric second order $B A B$ composition (III.2) and apply it for both splits. For the HO-split, we compute the harmonic part either 

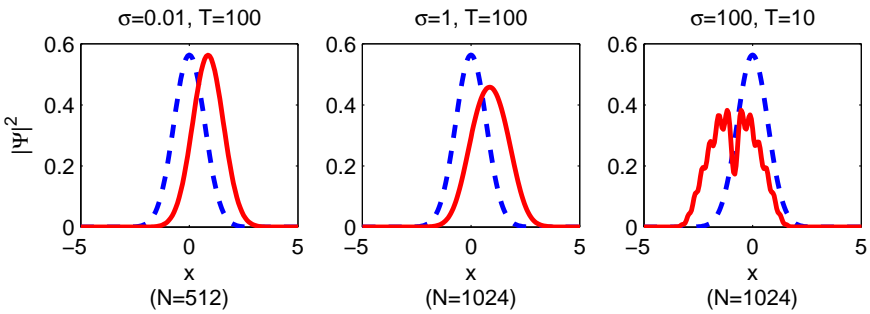

FIG. 2. Exact evolution at $t=T$ (solid line) from the initial conditions given by $\psi(x, 0)=\rho e^{-(x-1)^{2} / 2}$ (dashed line). The number of grid points is given by $N$.

with the decomposition (II.28) or in the Hermite basis (IV.1) with different numbers of basis terms.

The parameters, initial conditions and final times are taken as previously for Fig. 2. Given a splitting method $X$, we denote by $X_{F}, X_{H}$ and $X_{H} M$ its implementations with the F-split, the HO-split using the Hermite-Fourier method and the HO-split using $M$ Hermite basis functions in (IV.1), respectively. We measure the error versus the number of exponentials which can be considered proportional to the computational cost and plot the results in Fig. 3. As expected, HO-splits are advantagous if the system is close to a harmonic oscillator, i.e. for $\sigma=0.01,1$, and if the initial conditions are accurately approximated by a few terms in the Hermite expansion. On the other hand, for strong nonlinearities $\sigma=100$, the Hermite polynomial based HO split shows a very limited performance, c.f. the large number of basis terms in the right panel of Fig. 3. We stress that if this technique is used for nonlinearities the number of basis terms should be increased along the time integration and fixing it bounds the maximally achievable accuracy and its limit depends on the initial condition and the strength of the nonlinearity.

The Hermite-Fourier method proposed in this work (using the composition (II.28)) is clearly superior for weak perturbations and it keeps similar performance to the F-split for strong nonlinearities.

Finally, we analyze the performance of different higher order splitting methods which are useful when high accuracies are desired. The following methods (whose coefficients are collected in Table III for the convenience of the reader) are considered:

- $R K N_{6} 4$ (the 6-stage fourth-order method from [9]). This is a Partitioned Runge-Kutta-Nyström method and it is designed for the case where $[B,[B,[B, A]]]=0$, being the case for both the F-split and the HO-split.

- $N I_{4}(8,2)$ (the 4-stage $(8,2) B A B$ method from [1]). This method is addressed to perturbed systems. One expects a high performance if the contribution from $B$ is small.

- $N I_{5}(8,4)$ (the 5-stage $(8,4) B A B$ method from [1] $)$.

We analyze in Figures 4,6 the three problems specified in Fig. 3. In the upper panels, the Leap-Frog meth-
TABLE II. Coefficients for several splitting methods.

\begin{tabular}{ll}
\hline \hline \multicolumn{3}{c}{ The 6-stage 4th-order: $\mathrm{SRKN}_{6} 4$} \\
\hline \hline$b_{1}=0.0829844064174052$ & $a_{1}=0.245298957184271$ \\
$b_{2}=0.396309801498368$ & $a_{2}=0.604872665711080$ \\
$b_{3}=-0.0390563049223486$ & $a_{3}=1 / 2-\left(a_{1}+a_{2}\right)$ \\
$b_{4}=1-2\left(b_{1}+b_{2}+b_{3}\right)$ & $a_{4}=a_{3}, a_{5}=a_{2}, a_{6}=a_{1}$ \\
$b_{5}=b_{3}, b_{6}=b_{2}, b_{7}=b_{1}$ \\
\hline \hline \multicolumn{2}{c}{ The 4-stage $(8,2)$ method: $\mathrm{NI}_{4}$} \\
\hline \hline$b_{1}=1 / 20$ & $a_{1}=1 / 2-\sqrt{3 / 28}$ \\
$b_{2}=49 / 18$ & $a_{2}=1 / 2-a_{1}$ \\
$b_{3}=1-2\left(b_{1}+b_{2}\right)$ & $a_{3}=a_{2}, a_{4}=a_{1}$ \\
$b_{4}=b_{2}, b_{5}=b_{1}$ & \\
\hline \hline
\end{tabular}

\begin{tabular}{ll}
\hline \hline \multicolumn{1}{c}{ The 5-stage $(8,4)$ method: $\mathrm{NI}_{5}$} \\
\hline \hline$b_{1}=0.811862738544516$ & $a_{1}=-0.00758691311877447$ \\
$b_{2}=-0.677480399532169$ & $a_{2}=0.317218277973169$ \\
$b_{3}=1 / 2-\left(b_{1}+b_{2}\right)$ & $a_{3}=1-2\left(a_{1}+a_{2}\right)$ \\
$b_{4}=b_{3}, b_{5}=b_{2}, b_{6}=b_{1}$ & $a_{4}=a_{2}, a_{5}=a_{1}$ \\
\hline \hline
\end{tabular}

ods, LF, are compared with the second order $N I_{4}(8,2)$ methods. In the lower panels we compare the $R K N_{6} 4$ methods against the $(8,4)$ methods jointly with the best among the previous second order methods.

For a weak nonlinearity, when the system can be considered as a perturbed harmonic oscillator, we clearly observe that the HO-split is superior to the F-split. In this case, with a relatively small number of Hermite functions, it is possible to approach accurately the solution, but this procedure has a limited accuracy which can deteriorate along the time integration and depends on the initial conditions. In addition, the methods addressed to perturbed problems show the best performance: The $(8,2)_{H}$ method performs best among the compared when a relatively low accuracy is desired and the $(8,4)_{H}$ method takes its place for higher accuracies.

Figure 5 shows the results for $\sigma=1$. It is qualitatively similar to the previous case yet the HO-split does not outperform the plain F-split (II.21) as significantly as before. Nevertheless, it is important to observe that, again, the best result is obtained for the HO-split. Notice that a higher number of Hermite basis functions is necessary to achieve the same accuracy as the Hermite-Fourier decomposition.

Figure 6 shows the results for $\sigma=100$. The HO-split cannot be expected to be particularly useful because the system is far from being a harmonic oscillator. From Fig. 2, we expect a great number of Hermite basis functions to be required for a sufficiently accurate expansion. The results in Fig. 6] demonstrate this rather intuitive expectation, i.e. almost negligible precision despite the large number of basis terms $M=150$. Remarkably, the proposed HO decomposition does not show these limitations and reaches the precision of the F-split (II.21) because we are solving the harmonic potential exactly up to 

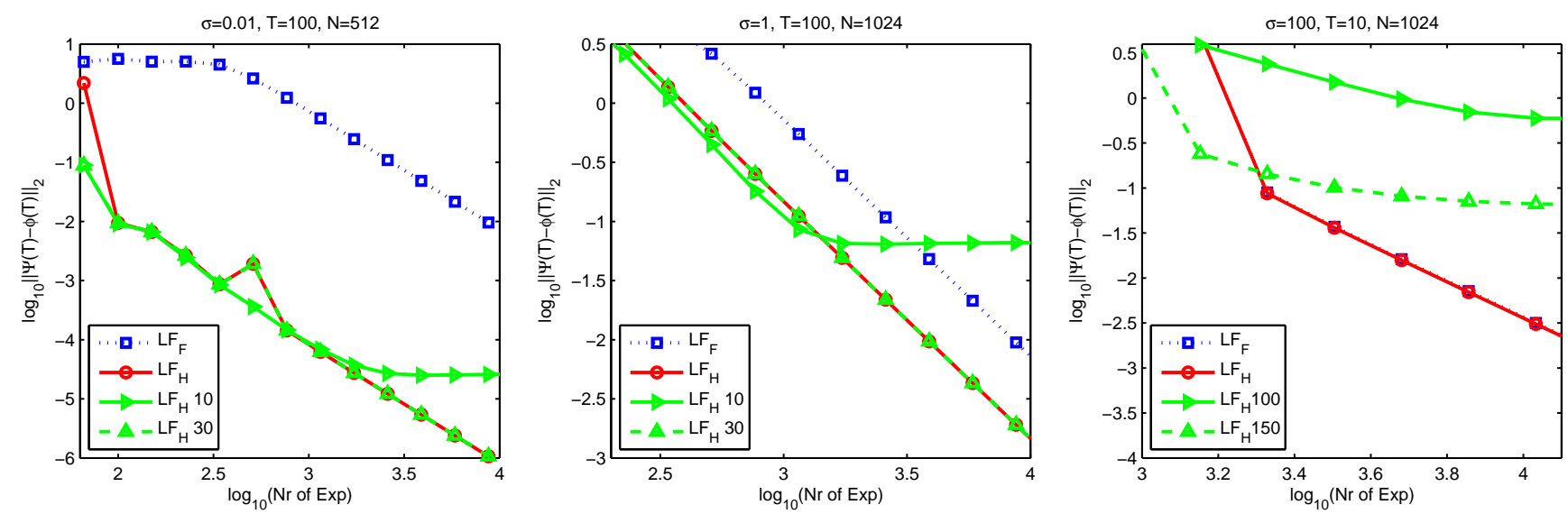

FIG. 3. Error versus the number of exponentials in logarithmic scale for different splittings for the Leap-Frog method.

spectral accuracy. For this problem, we observe that the $(8,2)$ method has the best performance when a relatively low accuracy is desired, the $(8,4)$ method shows the best performance for medium accuracies and the $R K N_{6} 4$ is the method of choice for higher accuracies.

\section{B. Time-dependent harmonic oscillator perturbed by weak quartic anharmonicity}

We consider now a harmonic oscillator with timedependent frequency and perturbed by a weak static quartic anharmonicity

$$
i \frac{\partial}{\partial t} \psi=\left(\frac{1}{2} p^{2}+\frac{1}{2} \omega^{2}(t) x^{2}\right) \psi+\varepsilon_{Q} \frac{1}{4} x^{4} \psi
$$

We first consider the case $\omega^{2}(t)=A(1+\epsilon \cos (w t))$ with $w=1 / 2, A=4, \epsilon=0.1, \varepsilon_{Q}=0.01$. As reference, we take a highly accurate numerical approximation as exact solution and restrict the spatial domain to $[-20,20]$ for all experiments in this subsection. We compare the Hermite-Fourier method with the plain Fourier split, since Hermite polynomials are not appropriate in a timedependent setting. For fast oscillating systems and if high accuracy is needed, the two-exponential fourth-order approximation of the harmonic oscillator (II.13) can be improved by taking, for example, a higher order Magnus expansion (III.12). As we have seen, the solution of

$$
i U^{\prime}=\left(\frac{1}{2} p^{2}+\frac{1}{2} \omega^{2}(t) x^{2}\right) U
$$

can be written as

$$
U(t, 0)=e^{-i \frac{t}{2}\left(\alpha x^{2}+\beta(x p+p x)+\gamma p^{2}\right)},
$$

and we have considered, for example, a sixth-order Magnus integrator [17] to approximate the evolution operator for one fractional time step, $a_{i} h$, i.e. $U\left(t, t+a_{i} h\right)$. This is equivalent to take in (IV.4 $t=a_{i} h$ and the parameters $\alpha, \beta, \gamma$ are given by:

$$
\begin{aligned}
\alpha & =\frac{1}{18}\left(5 \omega_{1}+8 \omega_{2}+5 \omega_{3}\right) \\
& +\frac{\left(a_{i} h\right)^{2}}{486}\left(\frac{17}{4}\left(\omega_{1}^{2}+\omega_{3}^{2}\right)+8 \omega_{2}^{2}+\omega_{1} \omega_{2}+\omega_{2} \omega_{3}-\frac{37}{2} \omega_{1} \omega_{3}\right), \\
\beta & =a_{i} h \sqrt{\frac{5}{3}}\left(\omega_{3}-\omega_{1}\right)\left(\frac{1}{12}+\frac{\left(a_{i} h\right)^{2}}{3240}\left(5 \omega_{1}+8 \omega_{2}+5 \omega_{3}\right)\right), \\
\gamma & =1+\frac{\left(a_{i} h\right)^{2}}{54}\left(\omega_{1}-2 \omega_{2}+\omega_{3}\right)
\end{aligned}
$$

with $\omega_{i}=\omega\left(t_{n}+c_{i} h\right), i=1,2,3$ and $c_{1}=1 / 2-\sqrt{15} / 10$, $c_{2}=1 / 2, c_{3}=1 / 2+\sqrt{15} / 10$, corresponding to a sixthorder Gaussian quadrature rule. The obtained operator is then decomposed according to Theorem $\llbracket .3$.

The results are given in Fig. 7 and corroborate the superiority of the HO split.

Another interesting example is given by an intense short pulse modeled via

$$
\omega(t)=w_{0}\left(1+\frac{A t}{\cosh ^{2}(B(t-2))}\right) .
$$

Varying the parameters $A$ and $B$, the pulse can be sharpened while keeping its time-average and hence its strength relative to the anharmonicity constant. Figure 8 shows the results obtained for a relatively slow variation of the harmonic potential, for the parameters: $w_{0}=4, A=0.25, B=2$. Again, the advantageousness of the presented decomposition can be appreciated. It is already noticeable, that the error introduced by the timedependence becomes dominant, and this effect increases for more rapidly varying potentials, e.g. for $B \gg 1$. In that case, higher order approximations of the Magnus expansion are necessary to maintain the benefits of the Hermite composition.

\section{CONCLUSIONS}

We have presented new Fourier methods for the numerical integration of perturbations to the time depen- 


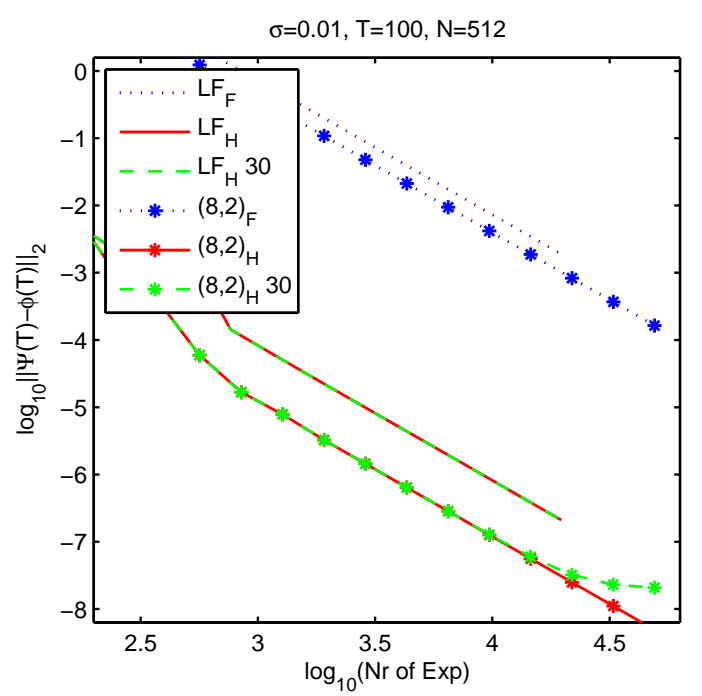

$\sigma=0.01, T=100, N=512$

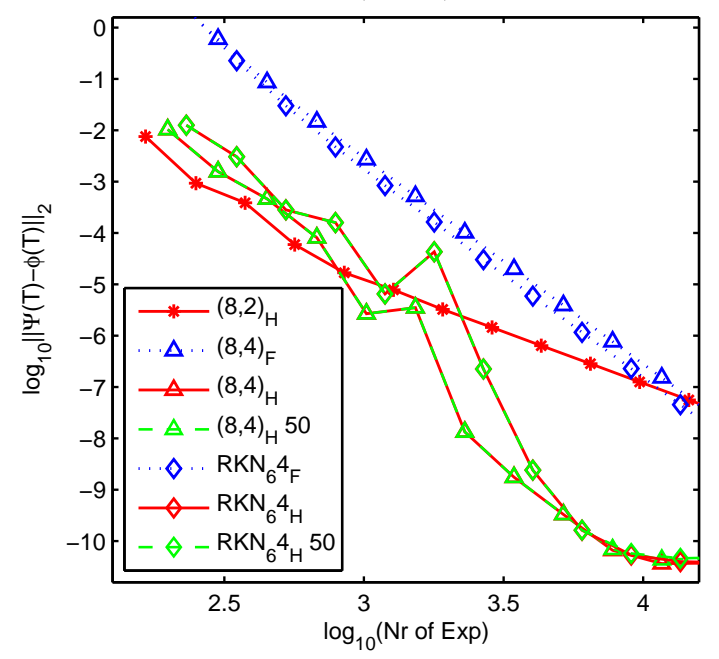

FIG. 4. Comparison of second order (upper panel) and fourth order (lower panel) methods for the different splittings and decompositions discussed in the text and $\sigma=0.01$.

dent harmonic oscillator which are useful for both both the Gross-Pitaevskii equation as well as for the linear Schrödinger equation. Fourier methods have shown a high performance in solving many different problems which can be split into the kinetic part and a remainder that is diagonal in the coordinate space. We have extended the Fourier methods to perturbations of the time-dependent harmonic potential, and refer to them as Hermite-Fourier methods. They solve the linear Schrödinger equation with a time-dependent harmonic potential to the desired order using corresponding Magnus expansions and up to the accuracy given by the spatial discretization. These methods are fast to compute since FFTs can be applied and show a high accuracy when the problem is a small perturbation of the har- monic potential. The methods presented in this work ex-
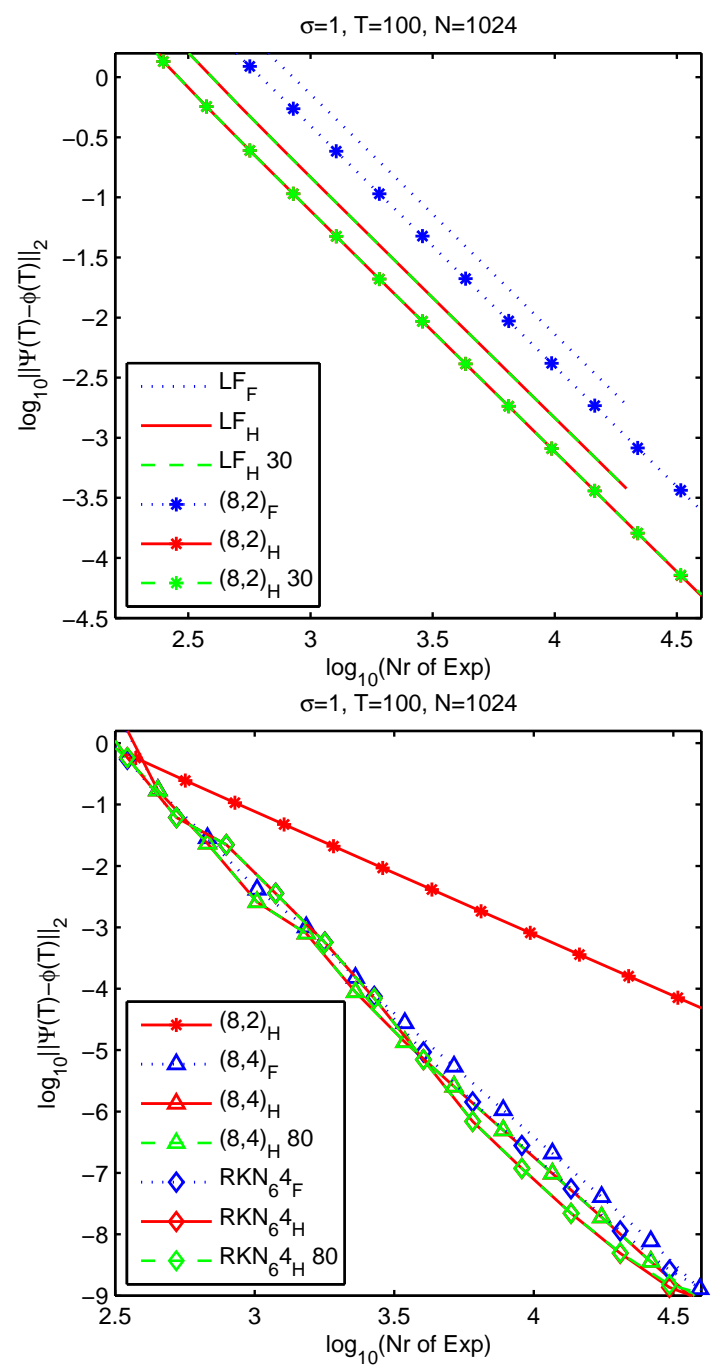

FIG. 5. Same as Fig. 4 for $\sigma=1$.

tend to perturbed harmonic potentials in linear quantum mechanics, c.f. section IVB, where it is straightforward to generalize the results to higher dimensions.

\section{ACKNOWLEDGMENTS}

We would like to acknowledge the referees for their careful reading and suggestions. The authors acknowledge the support of the Generalitat Valenciana through the project GV/2009/032. The work of S. Blanes has also been partially supported by Ministerio de Ciencia e Innovación (Spain) under the coordinated project MTM201018246-C03 (co-financed by the ERDF of the European Union). 

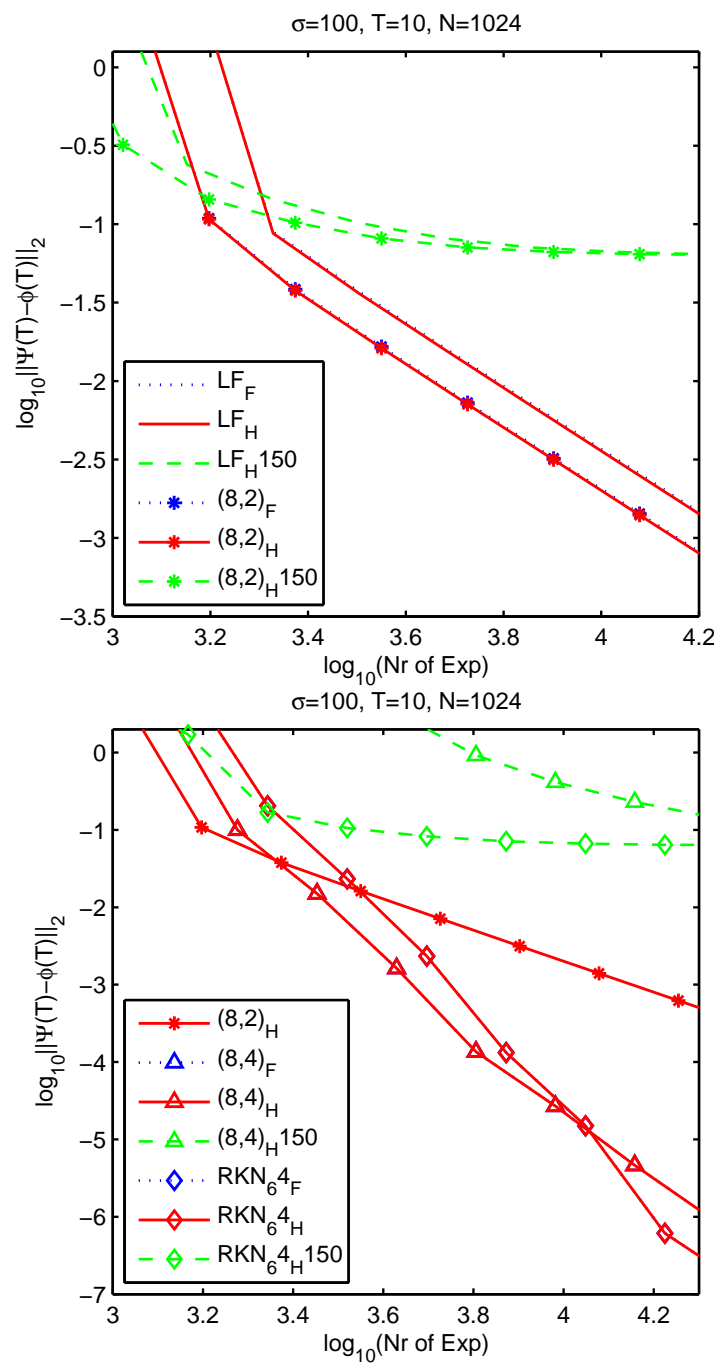

FIG. 6. Same as Fig. 4 for $\sigma=100$. F-splittings overlap the corresponding (red) Fourier-Hermite curves.

[1] W. Bao, D. Jaksch, P. Markowich, Numerical solution of the Gross-Pitaevskii equation for Bose-Einstein condensation, J. Comp. Phys. 187 (2003) 318-342.

[2] V.M. Pérez-García, X. Liu, Numerical methods for the simulation of trapped nonlinear Schrödinger systems, Appl. Math. Comp. 144 (2003) 215-235.

[3] M. Thalhammer, M. Caliari, and C. Neuhauser, Highorder time-splitting Hermite and Fourier spectral methods, J. Comput. Phys. 228 (2009), 822-832.

[4] M.H. Anderson, J.R. Ensher, M.R. Matthews, C.E. Wieman, and E.A. Cornell, Science 269, 198 (1995), C.C. Bradley, C.A. Sackett, J.J. Tollett, and R.G. Hulet, Phys. Rev. Lett. 75, 1687 (1995), K.B. Davis, M.-O. Mewes, M.R. Andrews, N.J. van Druten, D.S. Durfee, D.M. Kurn, and W. Ketterle, Phys. Rev. Lett. 75, 3969 (1995)
[5] C. J. Pethick, H. Smith, Bose-Einstein Condensation in Dilute Gases, Cambridge University Press (2001)

[6] W. Bao, J. Shen, A fourth-order time-splitting LaguerreHermite pseudospectral method for Bose-Einstein condensates, SIAM J. Sci. Comput. 26 (2005) 2010-2028.

[7] C.M. Dion, E. Cancés, Spectral method for the timedependent Gross-Pitaevskii equation with a harmonic trap, Phys. Rev. E 67 (2003) 046706.

[8] S. Blanes, F. Casas, and A. Murua, Splitting and composition methods in the numerical integration of differential equations, Bol. Soc. Esp. Math. Apl., 45 (2008), 87-143.

[9] S. Blanes and P. C. Moan, Practical symplectic partitioned Runge-Kutta and Runge-Kutta-Nyström methods, J. Comput. Appl. Math., 142 (2002), 313-330.

[10] E. Hairer, C. Lubich, and G. Wanner, Geometric Numerical Integration. Structure-Preserving Algorithms for 


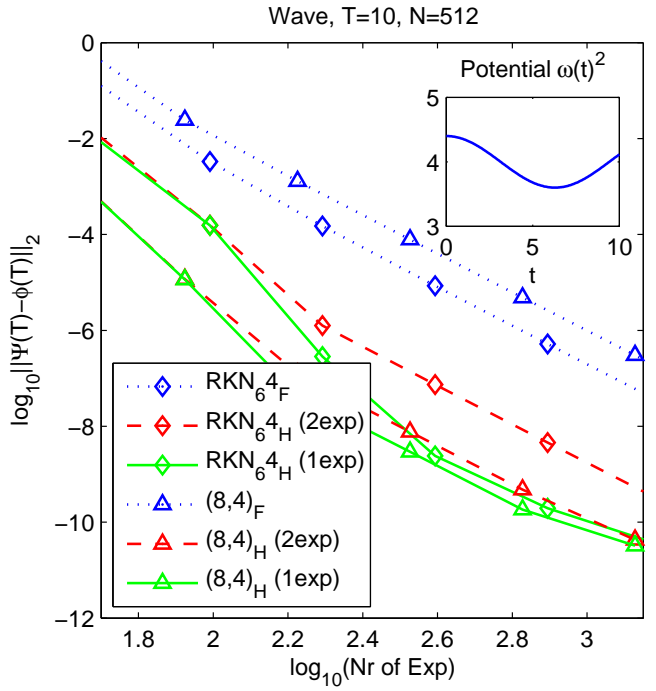

FIG. 7. Comparison of Fourier and Fourier-Hermite splittings for two fourth order methods. The (red) dashed line indicates the two-exponential approximation (II.13), the (green) solid line corresponds to the sixth-order Magnus approximation presented in the text (IV.4). The inset shows the evolution of the harmonic trap frequency $\omega(t)^{2}$ and the parameters used for the Hamiltonian are $w=1 / 2, A=4, \epsilon=0.1, \varepsilon_{Q}=0.01$

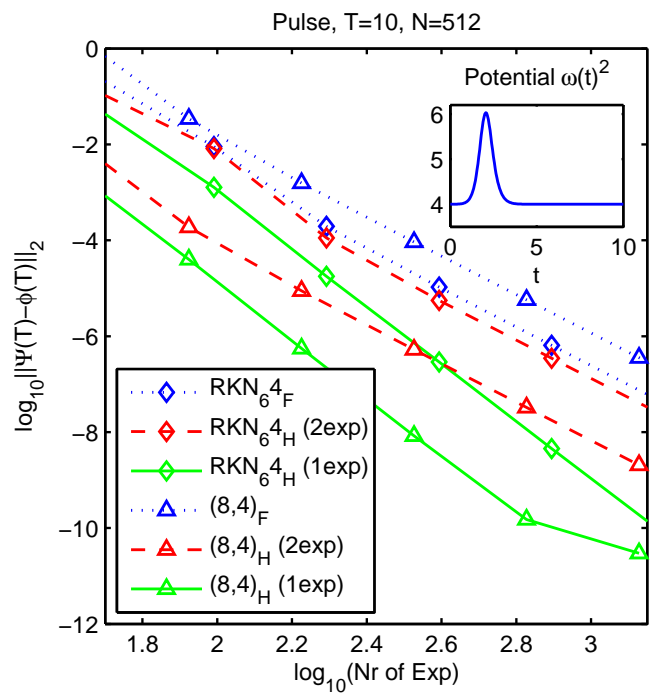

FIG. 8. Compare Fig. 7 The parameters used are $w_{0}=$ 4, $A=0.25, B=2$ with a small anharmonicity $\varepsilon_{Q}=0.01$
Ordinary Differential Equations, Springer Ser. Comput. Math. 31, Springer-Verlag, Berlin (2002).

[11] R. I. McLachlan, Composition methods in the presence of small parameters, BIT, 35 (1995), 258-268.

[12] R.I. McLachlan and R. Quispel, Splitting methods, Acta Numerica 11 (2002), 341-434.

[13] M. Suzuki, Fractal decomposition of exponential operators with applications to many-body theories and Monte Carlo simulations, Phys. Lett. A 146 (1990), 319-323.

[14] H. Yoshida, Construction of higher order symplectic integrators, Phys. Lett. A, 150 (1990), 262-268.

[15] S. Blanes, F. Diele, C Marangi,and S. Ragni, Splitting and composition methods for explicit time dependence in separable dynamical systems, J. Comput. Appl. Math. 235 (2010), 646-659.

[16] W. Magnus, On the exponential solution of differential equations for a linear operator. Commun. Pure Appl. Math., 7 (1954), 649-673.

[17] Blanes S, Casas F, Oteo JA, Ros J. The Magnus expansion and some of its applications, Phys. Rep., 470 (2009), $151-238$.

[18] J. Wei and E. Norman, Lie Algebraic Solution of Linear Differential Equations, J. Math. Phys. 4 (1963), 575-581.

[19] Since $F, G, E$ form a basis of the Lie algebra of the problem there exist functions $f_{i}(t)$ that correspond to the summation of the Magnus series. The functions can be obtained by solving a set of differential equations cf. 22

[20] S. Blanes and P.C. Moan, Splitting methods for the time-dependent Schrödinger equation, Phys. Lett. A, 265 (2000), 35-42.

[21] C. Lubich, From quantum to classical molecular dynamics: reduced models and numerical analysis. Zurich Lectures in Advanced Mathematics. European Mathematical Society (EMS), Zürich, 2008.

[22] R.M. Wilcox, Exponential operators and parameter differentiation in quantum physics, J. Math. Phys. 8 (1967), 962-982.

[23] S.A. Chin and E. Krotscheck, Fourth-order algorithm for solving the imaginary-time GrossPitaevskii equation in a rotating anisotropic trap, Phys. Rev. E 72 (2005), 036705.

[24] More precisely, the computational costs are due to a change of coordinates realized by the Fourier transform which is for this type of problem equivalent to the number of kinetic terms. 